\title{
Immune Status of COVID-19 Patients with Reference to SARS and MERS
}

\author{
Barani Karikalan $^{1 *}$ (D) and Hari Kumar Darnal ${ }^{2}$ \\ ${ }^{1}$ Department of Pathology, Perdana University, Selangor - 43400, Malaysia. ${ }^{2}$ Department of Pathology, Jeffrey \\ Cheah School of Medicine and Health Sciences, Monash University, Johor Bahru - 80100, Malaysia.
}

\begin{abstract}
During this global pandemic of COVID-19 infection, it became well known that morbidity and mortality is especially high at the extreme of life especially in certain racial or ethnic groups like Americans and Africans. This is presumed due to low immunity associated with other comorbid conditions like diabetes, hypertension, cardiovascular disease, obesity and metabolic syndrome. But the information available on the immune status of COVID-19 patients is limited. Attempts must be made to enhance our understanding of the immune status of COVID-19 patients by revisiting our knowledge on the immune mechanisms of already known coronaviruses such as SARS-CoV and MERS-CoV. Early elevation of the serum levels of pro-inflammatory cytokines observed in SARS and MERS infection suggests a possible same type of cytokine storm-mediated lung damage in COVID-19 patients too. Dysregulation of interferon-1 response and downstream cascade in initial innate immune response at virus entry point has been related to lethal pneumonia in COVID-19 patients. Adaptive response of increased CD8+ levels in COVID-19 patients seems to be useful in mild cases where it causes deteriorating effects in progressed severe disease patients resulting in destruction of type 2 pneumocytes hence inability to regenerate the alveolar epithelium. A phenomenon called cytokine storm activates violent immunological reactions in the lung tissue resulting in ARDS followed by multiple organ system damages in COVID-19 patients. Several immune evading mechanisms are thought to be employed by severe respiratory syndrome virus-2 (SARS-CoV-2) that might have resulted in its extremely increased contagiousness probably related with its frequent RNA mutations. Failure to develop adequate virus limiting immune reactions by some cured patients warrant monitoring of all recovered patients. This rapid mini review is aimed to enhance our knowledge of the immune status of COVID-19 infected patients with reference to SARS-CoV and MERS-CoV.
\end{abstract}

Keywords: Immune status, COVID-19, SARS-CoV, MERS-CoV, coronavirus

*Correspondence: baranisri@gmail.com; +60108148824

(Received: April 08, 2020; accepted: May 03, 2020)

Citation: Karikalan B, Darnal HK. Immune Status of COVID-19 Patients with Reference to SARS and MERS. J Pure Appl Microbiol. 2020;14(suppl 1):817-821. doi: 10.22207/JPAM.14.SPL1.18

(C) The Author(s) 2020. Open Access. This article is distributed under the terms of the Creative Commons Attribution 4.0 International License which permits unrestricted use, sharing, distribution, and reproduction in any medium, provided you give appropriate credit to the original author(s) and the source, provide a link to the Creative Commons license, and indicate if changes were made. 


\section{INTRODUCTION}

Even though the current information on the host innate immune status of COVID-19 patients is limited, our knowledge on SARS (Severe Acute Respiratory Syndrome) and MERS (Middle East respiratory syndrome) combined with preliminary findings of COVID-19 patients can provide us clues regarding the immune status of infected patients. Elevated total neutrophils and decreased total lymphocytes in COVID-19 patients presenting as mild and severe disease were found to be significantly different ${ }^{1}$. Elevated neutrophils and decreased lymphocyte significantly related to disease severity and death ${ }^{2}$. Early elevation in the serum levels of pro-inflammatory cytokines observed in SARS and MERS infection suggests a possible same type of cytokine storm-mediated lung damage in COVID-19 patients too ${ }^{3}$.

Innate immune response

When the viral particle enters the respiratory epithelial cells, its antigen is rendered to the antigen presentation cells (APC), which is the first step in the execution of the body's antiviral immune mechanism. Innate immune cells generally recognize the corona viral antigen by pathogen-associated molecular patterns (PAMPs). In the case of most RNA viruses including coronavirus, it is a well-known phenomenon that PAMPs which is the viral RNA or the intermediate structures during the viral replication processes such as dsRNA is recognized by various cytosolic RNA sensors and endosomal RNA receptors. This event of recognition is then followed by a number of down signaling pathways including nuclear translocation and transcription. In the nucleus, various transcription factors trigger the expression of Interferon 1 (IFN-1) along with other pro-inflammatory cytokines. This cascade of the event forms the first line of the defense system against the viral antigen at the point of entry ${ }^{4}$. Dysregulation in this IFN-1 response and downstream cascade has been related to lethal pneumonia in COVID-19 patients 5 .

\section{Adaptive immune response}

Th1 type immune reaction is the main component of adaptive immunity to most viral infections including coronaviruses. Like any usual acute viral infections, SARS and MERS also have the typical IgM and IgG antibodies in their antibody profile. IgM antibodies tailor out at the end of the $12^{\text {th }}$ week of viral entry whereas IgG antibodies last longer (6). Preliminary test results in COVID-19 patients have shown peak IgM at day 9 followed by a shift to IgG by $2^{\text {nd }}$ week (1). The latest reports show a reduced number of CD4+ and CD8+ lymphocytes in COVID-19 patients ${ }^{7}$. Along with lower lymphocytes counts, sever cases have elevated leukocytes counts and neutrophillymphocyte-ratio (NLR) and also decreased number of monocytes, eosinophils, and basophils ${ }^{8}$.

Previously, the initial rise in CD8+ cells has been associated with worse outcomes in the case of SARS and MERS. Details on whether such initial elevation of $C D 8$ + relates the severity of disease in COVID-19 patients' needs to be studied. Reduced CD8+ cells in severe cases of COVID-19 are thought to be involved in associated lung damage. Though protective, this CD8+ cells association with lung damage is attributed to the delayed or dysregulated immune response elicited by these cells. Therefore CD8+ T cell elevation, even though essential to keep the viral proliferation under control, has to be managed in order to prevent severe lung damage ${ }^{5}$.

\section{Cytokine storm}

Adult respiratory distress syndrome (ARDS) is known to be one of the main causes of death in COVID-19 patients ${ }^{9}$. ARDS was a common pathological mechanism of lung damage in SARS and MERS infections to ${ }^{10}$. ARDS has been associated with an important immunopathological mechanism known as a cytokine storm. SARS and MERS patients showed rapid elevation of many proinflammatory cytokines and chemokines such as IL-6, TNF, IFN- $\gamma$, and CCL5 are known to cause the cytokine storm ${ }^{9,11,12}$. Just like SARS and MERS, this cytokine storm activates violent immunological reactions in the lung tissue resulting in ARDS followed by multiple organ system damages in COVID-19 patients ${ }^{10}$. COVID-19 patients were seen to elicit increased levels of numerous pro-inflammatory cytokines as well as chemokines such as IFN-g, IL- 1 b, MCP-1, and IP10. Also, some severe cases of COVID-19 showed increased levels of G-CSF, TNF- $\alpha$, and MIP- $1 \mathrm{~A}^{13}$. Disease severity seems to correlate with the type of inflammatory cytokines found in COVID-19 patients. High levels of IL-2R and IL-6 seems to predict disease severity ${ }^{14}$. 


\section{Immune evasion}

Initial reaction by the immune system to the entry of viral particles is recognition of highly evolved viral structures called PAMPs via pattern recognition receptors (PRRs) of innate immune cells. SARS and MERS coronaviruses were seen to produce double-membrane vesicles that help escape this recognition, thereby allowing the replication of viral particles within these vesicles ${ }^{15}$. Likewise, both coronaviruses were seen to execute multiple mechanisms to interfere with signals leading to IFN-1 synthesis and hence further impede the downstream signaling cascade. These signal dampening strategies are known to be directly related to disease severity ${ }^{16}$. During the IFN-1 induction response, the SARS virus intrudes downstream signaling of RNA sensors either directly or indirectly. Indirect mechanisms include ubiquitination and degradation of RNA sensors and also by interfering with nuclear translocation. In addition to these mechanisms, the MERS virus uses additional strategies like modification of repressive histones. After the initial interferon secretion, SARS and MERS viruses were seen to become equipped with many mechanisms that interfere with interferon signaling ${ }^{17}$. Similar immune evading mechanisms might be employed by SARS-CoV-2 which might have resulted in its extremely increased contagiousness.

Mild vs severe disease

Comparing the distribution of various cellular compartments between control, mild and severe groups have been done. Increased proportions of T and NK cells were seen in the COVID-19 patients when compared with the control subjects. In comparison to mild cases, severe disease patients showed more macrophages and reduced the number of $T$ and NK cells. Data indicates not only increased recruitment of immune cells in the lungs of COVID-19 patients but also the increased immune cells correlated positively with disease severity. With regards to macrophage recruitment, severe disease patients showed a greater number of differentiated macrophage populations in addition to primitive monocyte populations. With regards to the CD8+ lymphocyte population, mild disease COVID-19 patients showed initial rise attributing to disease limitation. But some severe COVID-19 patients also showed a rise in CD8+ lymphocyte population which is considered to be because of activation induced by increased inflammation or a delayed immune reaction to the infection in severe COVID-19 patients ${ }^{18}$.

The discrepancy between the severity of cases have been seen to vary between different geographical location might be because of antibody dependent enhancement (ADE) because of previous exposure to other types of coronaviruses probably local viruses. ADE manipulates the immune reaction resulting in severe disease such as sustained inflammation, decreased lymphocytes and cytokine storm ${ }^{19}$.

Two-phase immune response

Immune responses in COVID-19 infection are seen to be in two phases. In the incubation phase and initial stages, specific adaptive immune reactions are responsible for virus limitation and to prevent progression to severe disease. The general health status of the patient along with the absence of genes that led to increased susceptibility to infections plays an important role in the progression of the disease from mild to severe stages. However, the absence of any of the above factor would result in disease progression especially involving organs with high numbers of ACE-2 receptors and possibly other unknown receptors. This viral proliferation triggers innate immune response contributed mainly by proinflammatory cytokines such as IL-2, IL-7, IL-10, IP-10, MCP-1, MIP-1A, G-CSF and TNF $\alpha$ secreted by macrophages and neutrophils in the lungs. Severe lung damage along with multiple organ failure is the main reason of death in COVID-19. Once damaged, the lung epithelial cells regeneration could occur from the type I pneumocytes. After enough lung damage the innate immune response may not be very helpful instead may cause more damage. Hence, at this stage inflammation needs to be controlled to prevent further lung damage and disease progression ${ }^{20}$.

Disturbingly, even after being cured and discharged from hospital, some COVID-19 patients returned to viral positivity and few of them again became symptomatic. This shows that some cured patients did not develop adequate virus limiting immune reactions. This warrants the monitoring of all recovered patients. Also, these patients may not respond to vaccines that are under developing stages for COVID-19, since they 
have difficulty in mounting an immune response against the viral particle. This phenomenon needs to be remembered while developing strategies for vaccines against COVID-19 infections ${ }^{21}$.

Immunosuppressed patients

Logically, patients with transplants are expected to have worse outcomes in coronavirus infections such as SARS, MERS and COVID-19. But no known worsening case of SARS was reported in immunosuppressed patients ${ }^{22}$. Previous data on SARS and MERS along with recent reports on COVID-19 shows no evidence of immunosuppression as a risk factor for virus acquisition in all three coronaviruses. No fatality or rise in morbidity and mortality has been attributed to immunosuppression in any form ${ }^{23}$. A recent report on the effect of cancer chemotherapy on COVID-19 patients seems to be largely related to other associated comorbidities more than cancer therapy itself ${ }^{24}$.

\section{CONCLUSION}

Understanding of the immune status of COVID-19 patients must be attempted with all the available limited research data and known knowledge on other coronaviruses like SARS$\mathrm{CoV}$ and MERS-CoV. This understanding must be remembered while devising and formulating strategies for development of vaccines and therapies for COVID-19 infection to enhance the outcome. This rapid mini review is an attempt at understanding the immune status of COVID-19 infected patients with reference to SARS-CoV and MERS-CoV viruses. This may help in follow up and vaccine development in very near future.

\section{ACKNOWLEDGMENTS}

Authors would like to thank Perdana University e-library resources for their services.

\section{CONFLICT OF INTEREST}

The listed author(s) declare no conflict of interest in any capacity, including competing or financial.

\section{AUTHORS' CONTRIBUTION}

All listed author(s) have made a substantial, direct and intellectual contribution to the work, and approved it for publication.

\section{FUNDING}

None.

\section{ETHICS STATEMENT}

This article does not contain any studies with human participants or animals performed by any of the authors.

\section{AVAILABILITY OF DATA}

Not applicable.

\section{REFERENCES}

1. Zhou P, Yang XL, Wang XG, et al. A pneumonia outbreak associated with a new coronavirus of probable bat origin. Nature. 2020;579(7798):270-273.

2. Wu F, Zhao S, Yu B, et al. A new coronavirus associated with human respiratory disease in China [published correction appears in Nature. 2020;580(7803): E7]. Nature. 2020;579(7798):265-269.

3. Huang C, Wang Y, Li X, et al. Clinical features of patients infected with 2019 novel coronavirus in Wuhan, China [published correction appears in Lancet. 2020 Jan 30]. Lancet. 2020;395(10223):497-506. https://doi. org/10.1016/S0140-6736(20)30183-5

4. de Wit E, van Doremalen N, Falzarano D, Munster VJ. SARS and MERS: recent insights into emerging coronaviruses. Nat Rev Microbiol. 2016;14(8):523-534. https://doi.org/10.1038/nrmicro.2016.81

5. Prompetchara E, Ketloy C, Palaga T. Immune responses in COVID-19 and potential vaccines: Lessons learned from SARS and MERS epidemic. Asian Pac J Allergy Immunol. 2020;38:1-9.

6. Li G, Chen X, Xu A. Profile of specific antibodies to the SARS-associated coronavirus. N Engl J Med. 2003;349(5):508-9. https://doi.org/10.1056/ NEJM200307313490520

7. Li X, Geng M, Peng Y, Meng L, Lu S. Molecular immune pathogenesis and diagnosis of COVID-19. J Pharm Analysis. 2020;10(2):102-108. https://doi. org/10.1016/j.jpha.2020.03.001

8. Qin C, Zhou L, Hu Z, et al. Dysregulation of immune response in patients with COVID-19 in Wuhan, China [published online ahead of print, 2020 Mar 12]. Clin Infect Dis. 2020;ciaa248. https://doi.org/10.2139/ ssrn.3541136

9. Huang C, Wang Y, Li X, et al. Clinical features of patients infected with 2019 novel coronavirus in Wuhan, China. Lancet. 2020;395:497-506. https://doi.org/10.1016/ S0140-6736(20)30183-5

10. Xu Z, Shi L, Wang Y, et al. Pathological findings of COVID-19 associated with acute respiratory distress syndrome [published correction appears in Lancet Respir Med. 2020 Feb 25]. Lancet Respir Med. 2020;8(4):420-422. https://doi.org/10.1016/S22132600(20)30076-X

11. Chen L, Liu HG, Liu W, et al. Analysis of clinical features of 29 patients with 2019 novel coronavirus pneumonia. ZhonghuaJie He He Hu Xi ZaZhi. 2020;43(3):203-208.

12. Williams $A E$, Chambers RC. The mercurial nature of 
neutrophils: still an enigma in ARDS? Am J Physiol Lung Cell Mol Physiol. 2014;306(3):L217-L230. https://doi. org/10.1152/ajplung.00311.2013

13. Cameron MJ, Bermejo-Martin JF, Danesh A, Muller $M P$, Kelvin DJ. Human immunopathogenesis of severe acute respiratory syndrome (SARS). Virus Res. 2008; 133(1):13-19. https://doi.org/10.1016/j. virusres.2007.02.014

14. CK Min, S Cheon, NY Ha, et al. Comparative and kinetic analysis of viral shedding and immunological responses in MERS patients representing a broad spectrum of disease severity. Sci Rep. 2016:6:25359. https://doi. org/10.1038/srep25359

15. Snijder EJ, van der Meer $Y$, Zevenhoven-Dobbe $J$, et al. Ultrastructure and origin of membrane vesicles associated with the severe acute respiratory syndrome coronavirus replication complex. J Virol. 2006;80(12):5927-5940. https://doi.org/10.1128/ JVI.02501-05

16. Channappanavar R, Perlman S. Pathogenic human coronavirus infections: causes and consequences of cytokine storm and immunopathology. Semin Immunopathol. 2017;39(5):529-539. https://doi. org/10.1007/s00281-017-0629-x

17. Kindler $E$, Thiel $V$, Weber $F$. Interaction of SARS and MERS Coronaviruses with the Antiviral Interferon Response. Adv Virus Res. 2016;96:219-43. https://doi. org/10.1016/bs.aivir.2016.08.006

18. Liao M, Liu Y, Yuan J, et al. The landscape of lung bronchoalveolar immune cells in COVID-19 revealed by single-cell RNA sequencing. $B M J$. The preprint server for health sciences. medrxiv;2020.02.23.20026690v1, [Preprint]. https:// doi.org/10.1101/2020.02.23.20026690

19. Tetro JA. Is COVID-19 receiving ADE from other coronaviruses? Microbes Infect. 2020;22(2):72-73. https://doi.org/10.1016/j.micinf.2020.02.006

20. $\mathrm{Xu} \mathrm{Z}$, Shi L, Wang $\mathrm{Y}$, et al. Pathological findings of COVID-19 associated with acute respiratory distress syndrome [published correction appears in Lancet Respir Med. 2020 Feb 25]. Lancet Respir Med. 2020;8(4):420-422. https://doi.org/10.1016/S22132600(20)30076-X

21. Shi $Y$, Wang $Y$, Shao $C$. et al. CoVID-19 infection: the perspectives on immune responses. Cell Death Differ. 2020;27:1451-1454. https://doi.org/10.1038/s41418020-0530-3

22. Hui DS, Azhar El, Kim YJ, Memish ZA, et al. Middle East respiratory syndrome coronavirus: risk factors and determinants of primary, household, and nosocomial transmission. Lancet Infect Dis. 2018;18:e217-e227. https://doi.org/10.1016/S1473-3099(18)30127-0

23. Liang W, Guan W, Chen R, Wang W, et al. Cancer patients in SARS-CoV-2 infection: a nationwide analysis in China. Lancet Oncol. 2020; 21:335-337. https://doi. org/10.1016/S1470-2045(20)30096-6

24. D'Antiga L. Coronaviruses and immunosuppressed patients. The facts during the third epidemic. Liver Transpl. 2020 Mar 20. doi: 10.1002/lt.25756. [Epub ahead of print] https://doi.org/10.1002/It.25756 